\title{
InP-based Lattice-Matched InGaAsP and Strain-Compensated InGaAs/InGaAs Quantum Well Cells for Thermophotovoltaic Applications
}

Carsten Rohr, Paul Abbott, James P. Connolly, and Keith W. J. Barnham* Experimental Solid State Physics, Blackett Laboratory, Imperial College London, SW7 2AZ, U.K.

Massimo Mazzer

CNR-IMM, Sez. di Lecce, via Arnesano, 73100 Lecce, Italy

Lucia Nasi

CNR-IMEM Sez. di Parma, Parco Area delle Scienze 37/A, 43010 Fontanini- Parma, Italy

Chris Button and John S. Roberts

EPSRC National Centre for III-V Technologies, Sheffield S1 3JD, U.K.

\author{
Graham Clarke \\ IQE Ltd., Cardiff CF3 OEG, U.K. \\ Ravin Ginige \\ NMRC, University College, Cork, Eire
}

(Dated: February 1, 2005) 


\begin{abstract}
Quantum Well Cells (QWCs) for thermophotovoltaic (TPV) applications are demonstrated in the InGaAsP material system lattice-matched to the InP substrate and strain-compensated InGaAs/InGaAs QWCs also on InP substrates. We show that lattice-matched InGaAsP QWCs are very well suited for TPV applications such as with Erbia selective emitters. QWCs with the same effective bandgap as a bulk control cell show a better voltage performance. Hence a QWC can improve the efficiency of a bulk control cell as we demonstrate. A major advantage of QWCs is that the bandgap can be engineered by changing the well thickness and varying the composition to fit the illuminating spectrum. This is relatively straight-forward in the lattice-matched InGaAsP system. This approach can be extended to longer wavelengths by using strain-compensation techniques, achieving bandgaps as low as $0.62 \mathrm{eV}$ that cannot be achieved with lattice-matched bulk material. We show that strain-compensated QWCs have voltage performances that are at least as good as, if not better than, expected from bulk control cells.
\end{abstract}

*Electronic address: k. barnham@imperial.ac.uk 


\section{INTRODUCTION}

In a Quantum Well Cell (QWC) nanometre thick layers of low bandgap material are introduced into the intrinsic region of a p-i-n diode in order to increase the absorption of a photovoltaic (PV) cell [1]. The absorption threshold or effective bandgap can be tuned by varying the material composition of these quantum wells (QWs) and, because of the quantum confinement, by changing their thickness. For cells based on the InP substrate, a wide range of lattice-matched materials is available, $\mathrm{In}_{1-\mathrm{x}} \mathrm{Ga}_{\mathrm{x}} \mathrm{As}_{\mathrm{y}} \mathrm{P}_{1-\mathrm{y}}$ with $\mathrm{x} \approx 0.47 \mathrm{y}$, where the bandgap can be varied between 0.9 and $1.7 \mu \mathrm{m}$, with $\operatorname{In}_{0.53} \mathrm{Ga}_{0.47} \mathrm{As}$ having the lowest possible bandgap of this lattice-matched material system.

The absorption can be extended if the QWs are strained to the substrate. But only a few QWs can be grown before dislocations form and the device quality deteriorates due to non-radiative recombination [2]. This restriction can be overcome by strain compensating QWs and barriers. In principle, an unlimited number of periods can be grown without strain relaxation if the stress is minimised by appropriate strain-compensation $[3,4]$.

In thermophotovoltaics (TPV) [5, 6], heat radiation is converted into electricity, as opposed to sunlight in a conventional PV cell. The source is at a lower temperature for TPV applications, typically around $1500-2000 \mathrm{~K}$, as opposed to the $5800 \mathrm{~K}$ of the sun. The peak of a lower temperature black-body spectrum is at a longer wavelength, hence lower band-gap semiconductors offer a better spectral match. It is also possible to shape the illuminating spectrum, for example by introducing selective emitters between the source and the devices to obtain a narrow band spectrum [7], which increases the conversion efficiency when matched to the cell. Such selective emitters are often based on rare-earth elements, such as Erbium, Thulium or Holmium with peak emission of about 1.5, 1.8 and $2.1 \mu \mathrm{m}$, respectively $[7]$.

In this paper we study and analyse lattice-matched InGaAsP QWCs suitable for an Erbium-based emitter (Section III A), and strain-compensated InGaAs/InGaAs QWCs for a Thulium-based emitter (Section III B). A more general review of QWCs for TPV applications is given in Ref. [8]. 
TABLE I: Sample description of a typical lattice-matched quaternary QWC, in this case sample MR1178. Q1.1 and Q1.6 represent the quaternary InGaAsP material with a bulk absorption edge of about 1.1 and $1.6 \mu \mathrm{m}$ respectively.

\begin{tabular}{llccccc}
\hline \hline & Layer & Repeats & Thickness $[\AA]$ & Material & Doping & Conc. $\left[\mathrm{cm}^{-3}\right]$ \\
\hline \multirow{6}{*}{ Cap/Contact } & 1 & 1000 & InGaAs & $\mathrm{p}$ & $3 \times 10^{18}$ \\
& Emitter & 1 & 1500 & $\mathrm{InP}$ & $\mathrm{p}$ & $8 \times 10^{17}$ \\
& Spacer & 1 & 2500 & $\mathrm{InP}$ & $\mathrm{i}$ & \\
Lattice- & Barrier & 60 & 70 & $\mathrm{Q} 1.1$ & $\mathrm{i}$ & \\
MQtched & Well & 60 & 100 & $\mathrm{Q} 1.6$ & $\mathrm{i}$ & \\
& Barrier & 1 & 70 & $\mathrm{Q} 1.1$ & $\mathrm{i}$ & \\
& Base/Buffer & 1 & 5000 & $\mathrm{InP}$ & $\mathrm{n}$ & $3 \times 10^{18}$ \\
\hline \hline
\end{tabular}

\section{EXPERIMENTAL}

The lattice-matched InGaAsP devices were grown on InP substrates by metal-organic vapour-phase epitaxy (MOVPE) at the EPSRC National Centre for III-V Technologies in Sheffield, UK. A typical sample with a multi-quantum well (MQW) region is given in Table I. The layers were grown on epi-ready (100) InP substrates, $n$-type with sulphur, and usually with a misorientation of 0.35 degrees off to (110). The $p$ - and $n$-type layers are InP, whereas the $\sim 1 \mu \mathrm{m}$ wide intrinsic region is made of the quaternary Q1.1 (where the $\mathrm{Q}$ value indicates the bulk absorption edge in microns) with a composition of $y \approx 0.36$ resulting in a bulk absorption edge of $1.1-1.15 \mu \mathrm{m}$. Incorporated into this $i$-region are $60 \mathrm{QWs}$ of $100 \AA$ width made of the quaternary Q1.6 with a composition of $y \approx 0.86$ equivalent to a bulk absorption edge of about $1.6 \mu \mathrm{m}$, but due to the confinement the effective absorption edge is closer to $1.5 \mu \mathrm{m}$. A $0.2 \mu \mathrm{m}$ wide spacer region is introduced between the $i$ - and $p$-region to reduce Zn dopant diffusion into the QWs [9]. The top InGaAs cap layer serves for contacting the device. When an anti-reflection (AR) coating is applied, the cap is etched off in the optical window.

Some strain-compensated samples were also grown in Sheffield (prefix 'MR'), but most were grown by IQE Ltd, Cardiff, Wales (prefix 'E'), also by MOVPE, such as E1055 shown in Table II. 
TABLE II: Sample description of strain-compensated QWC E1055.

\begin{tabular}{clccccc}
\hline \hline & Layer & Repeats & Thickness $[\AA]$ & Material & Doping & Conc. $\left[\mathrm{cm}^{-3}\right]$ \\
\hline & Cap/Contact & 1 & 3000 & $\mathrm{InGaAs}$ & $\mathrm{p}$ & $1 \times 10^{19}$ \\
& Emitter & 1 & 3000 & $\mathrm{InP}$ & $\mathrm{p}$ & $2 \times 10^{18}$ \\
& Spacer & 1 & 2000 & $\mathrm{InP}$ & $\mathrm{i}$ & \\
Strain- & Barrier & 40 & 89 & $\mathrm{In}_{0.45} \mathrm{Ga}_{0.55} \mathrm{As}$ & $\mathrm{i}$ & \\
compensated & Well & 40 & 80 & $\mathrm{In}_{0.74} \mathrm{Ga}_{0.26} \mathrm{As}$ & $\mathrm{i}$ & \\
MQW & Barrier & 40 & 89 & $\mathrm{In}_{0.45} \mathrm{Ga}_{0.55} \mathrm{As}$ & $\mathrm{i}$ & \\
& Buffer & 1 & 200 & $\mathrm{InP}$ & $\mathrm{i}$ & \\
& Base & 1 & 5000 & $\mathrm{InP}$ & $\mathrm{n}$ & $1 \times 10^{18}$ \\
\hline \hline
\end{tabular}

Test devices were processed into $1 \mathrm{~mm}$ diameter mesas with a contact ring leaving an optical window of $600 \mu \mathrm{m}$ diameter.

A Keithley 238 Source-Measurement Unit was used for current-voltage (I-V) measurements in the dark and under illumination. The temperature of the sample, which was kept constant at $25^{\circ} \mathrm{C}$ using a Peltier plate, was monitored with a Keithley 142 Digital MultiMeter and a platinum resistance thermometer. An Oriel quartz halogen tungsten lamp was used as light source with a stabilised DC power supply and a fibre lead, approximating a $3000 \mathrm{~K}$ black-body spectrum. The power density was set to a constant level using a calibration cell.

The quantum efficiency (QE) was measured using a Bentham M300 0.3 m monochromator with a $100 \mathrm{~W}$ tungsten lamp as a light source and a Bentham current stabilised filament lamp power supply 505. The photocurrent is measured with a lock-in technique using a Stanford Research Systems SR510 Lock-in Amplifier with a Bentham 218 variable frequency optical chopper at a frequency of about $187 \mathrm{~Hz}$. Two such set-ups were used, one for the visible range (400-1100 nm) with a calibrated Si detector, and another one for the infrared range (850-2000 nm) with calibrated InGaAs (850-1650 nm) and extended InGaAs (1300-2000 nm) detectors from Hamamatsu. 


\section{RESULTS}

\section{A. Lattice-matched InGaAsP QWCs}

Several lattice-matched InGaAsP QWCs were grown similar to MR1178 (see Table I) and a nominally identical sample MR1180 which had an additional etch-stop layer between the base and the substrate for front contacting. For comparison, control samples without QWs but otherwise the same as in Table I (i.e. with the same total i-region width) were grown: a barrier control MR1088, where the whole i-region consists of the same barrier material Q1.1; and a well control MR1091 where the i-region consists of a quaternary material composition so that the effective absorption edge is the same as in the QWC (i.e. material with a slightly wider bandgap than Q1.6 to compensate for the confinement in the QWs). Hence samples MR1088 and MR1091 are double hetero-structures. As one can see in Fig. 1, samples MR1091 and MR1180 have exactly the same absorption edge, while MR1178 extends somewhat further. These samples are very well suited for TPV applications with Erbium-based selective emitters which have a peak at about $1500 \mathrm{~nm}$ (also indicated in Fig. 1).

One would expect the voltage performance to be determined by the bandgap of the QW material. The the most challenging situation is to compare samples with the same effective threshold, as in this set of samples. Although the absorption threshold is identical for MR1091 and MR1180 (see Fig. 1), the dark current density of the QWC is significantly lower than that of the well control as shown in Fig. 2. The QWC MR1178 shows even better material quality having a similarly low dark current as QWC MR1180 while the absorption is extended to slightly longer wavelengths.

Barnham et al. have demonstrated a voltage enhancement of QWCs compared to their bulk control cells in the lattice-matched material systems AlGaAs/GaAs, GaInP/GaAs, $\mathrm{InP} / \mathrm{InGaAs}$ [10]. However, in those cases the control cells were made of the same material as the QWs, which means that the bulk control cells have slightly lower effective bandgaps due to the confinement in the QWs. Here we demonstrate that a QWC with the same effective bandgap as a bulk control (Fig. 1) shows a lower dark current (Fig. 2) and a voltage enhancement (Fig. 3).

This is further evidence that QWCs have a better dark current than expected from their absorption threshold [10]. In Fig. 4, this is shown to be true also for the material 


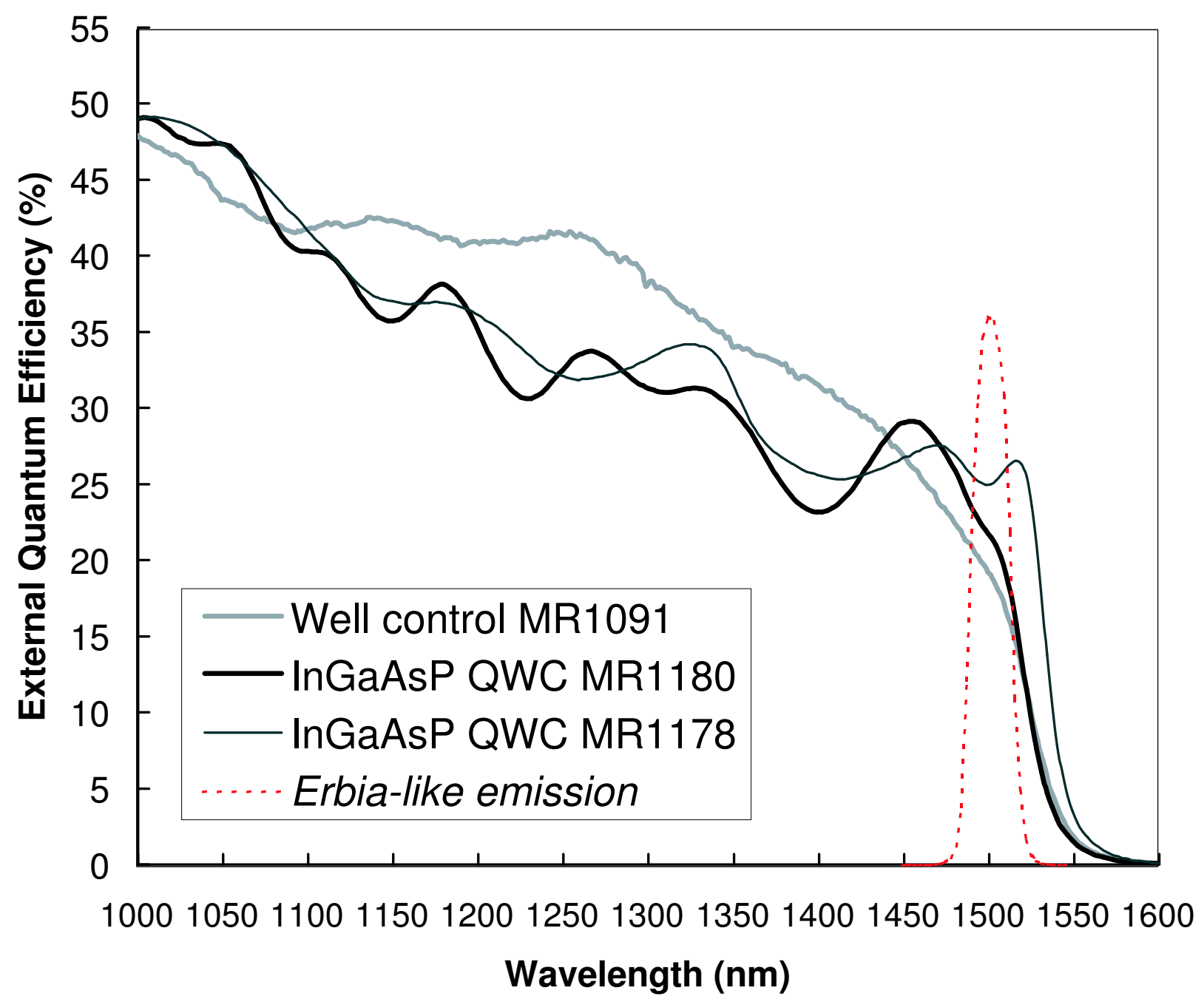

FIG. 1: External Quantum Efficiency of QWCs MR1180, MR1178, compared with well control MR1091 (all non-AR). The position of an Erbia peak is also indicated (arbitrary intensity).

system $\mathrm{In}_{1-\mathrm{x}} \mathrm{Ga}_{\mathrm{x}} \mathrm{As}_{\mathrm{y}} \mathrm{P}_{1-\mathrm{y}}$ on InP. One might expect the dark current (for example at a typical operating point such as a bias of $0.4 \mathrm{~V}$ ) of the QWCs to lie on a line connecting the two homogenous bulk controls InP and InGaAs (Fig. 4), including an InGaAs monolithic interconnected module (MIM) [11]. However, all the QWCs have a much lower dark current than that by about two orders of magnitude. The dark current depends to some degree on the number of QWs, particularly for a small number of QWs, but here we consider large numbers of QWs as all the lattice-matched QWCs have 60 QWs. Also included in Fig. 4 are double hetero-structures such as MR1091 and MR1088 which are described above. 


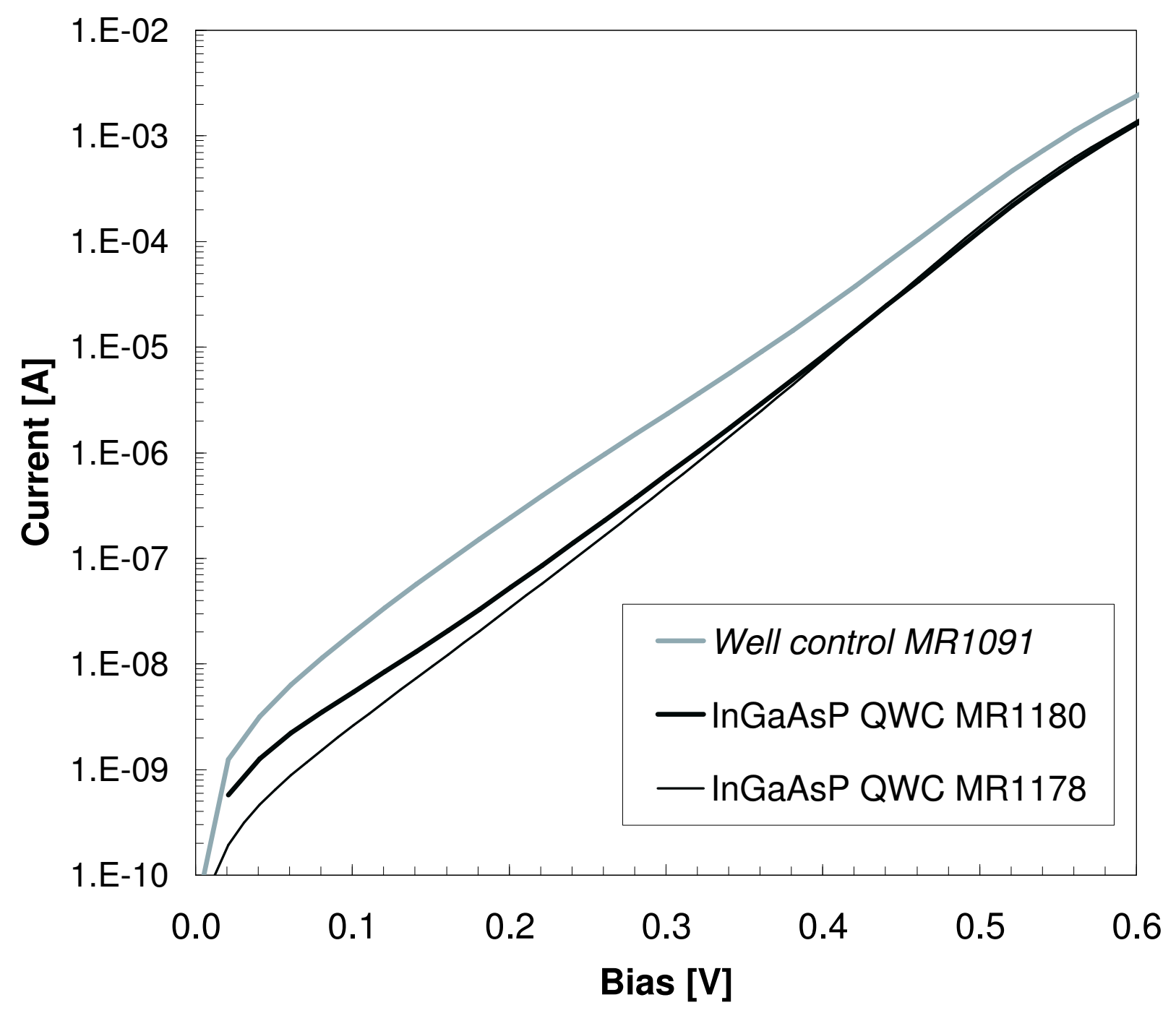

FIG. 2: Dark current densities of QWCs MR1180, MR1178, compared with well control MR1091.

Although, double hetero-structures have a much lower dark current than the homogenous controls, their dark current is not as low as most QWCs. This shows that InGaAsP QWCs have an improved voltage performance and hence a clear advantage over homogenous bulk control cells and even over double hetero-structures.

The I-V characteristics under illumination from the $3000 \mathrm{~K}$ black body source are shown for some of the cells in Fig. 3. Although the absorption thresholds of MR1091 and MR1180 are identical, the well control MR1091 has a slightly higher short-circuit current $\mathrm{I}_{\mathrm{SC}}$ than the QWC MR1180 because it has a slightly higher QE in the range 1100-1450 nm (see Fig. 1) which gives rise to a considerable contribution under the broad illuminating spec- 


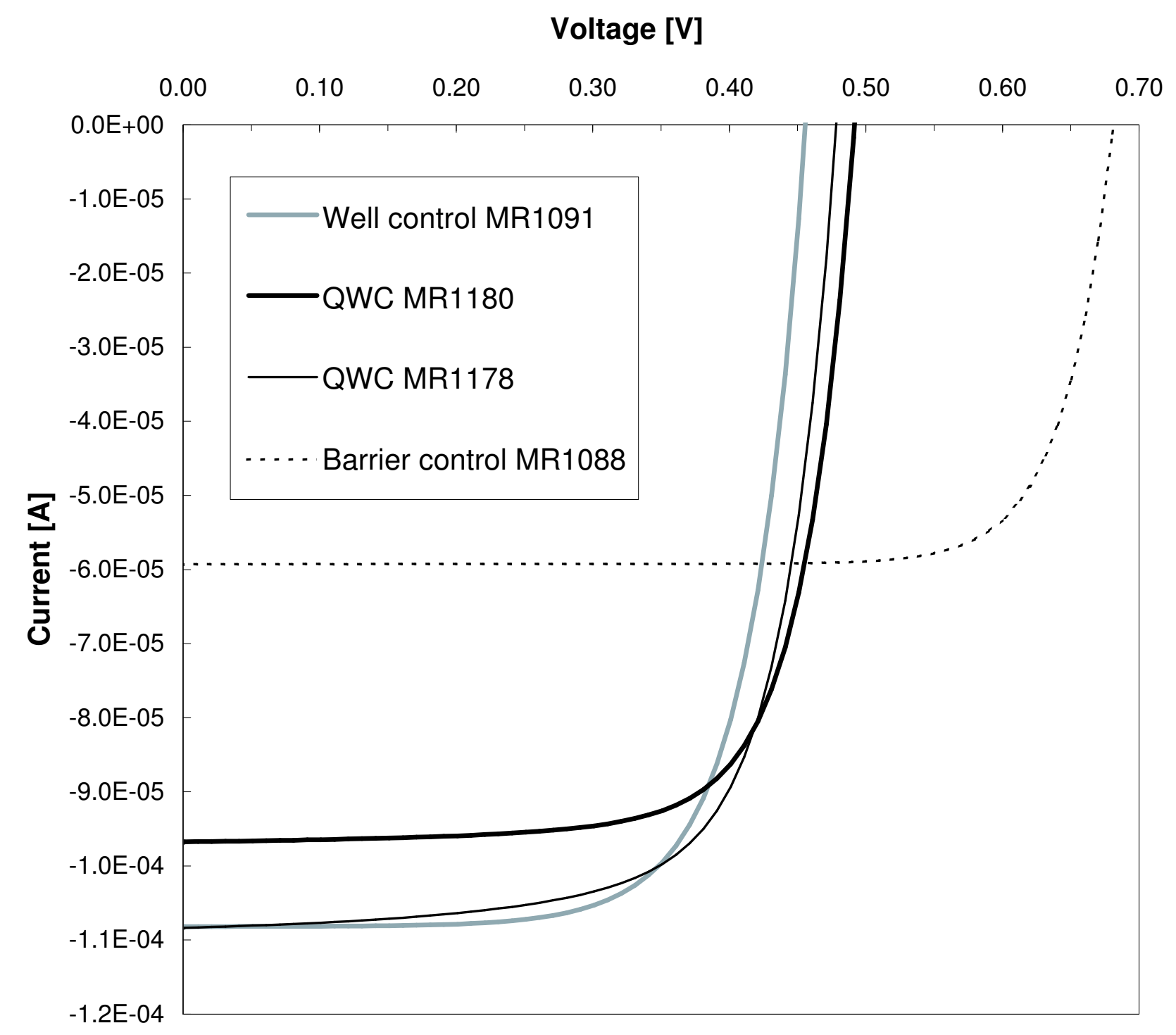

FIG. 3: I-V curves of QWCs MR1180, MR1178, well control MR1091 and barrier control MR1088 under $3000 \mathrm{~K}$ blackbody illumination.

trum. However, this effect should be much less under a narrow band spectrum, such as an Erbia selective emitter, where the QWC MR1180 is expected to outperform the well control MR1091. Even though $I_{\mathrm{sc}}$ is smaller for MR1180 than for MR1091, the $V_{\mathrm{oc}}$ is significantly higher for MR1180. It shows that the reduced dark current leads to a higher $V_{\text {oc }}$.

The QWC MR1178 shows an improvement in performance over both the barrier control (MR1088) and the well control (MR1091) even under this broad illuminating spectrum 


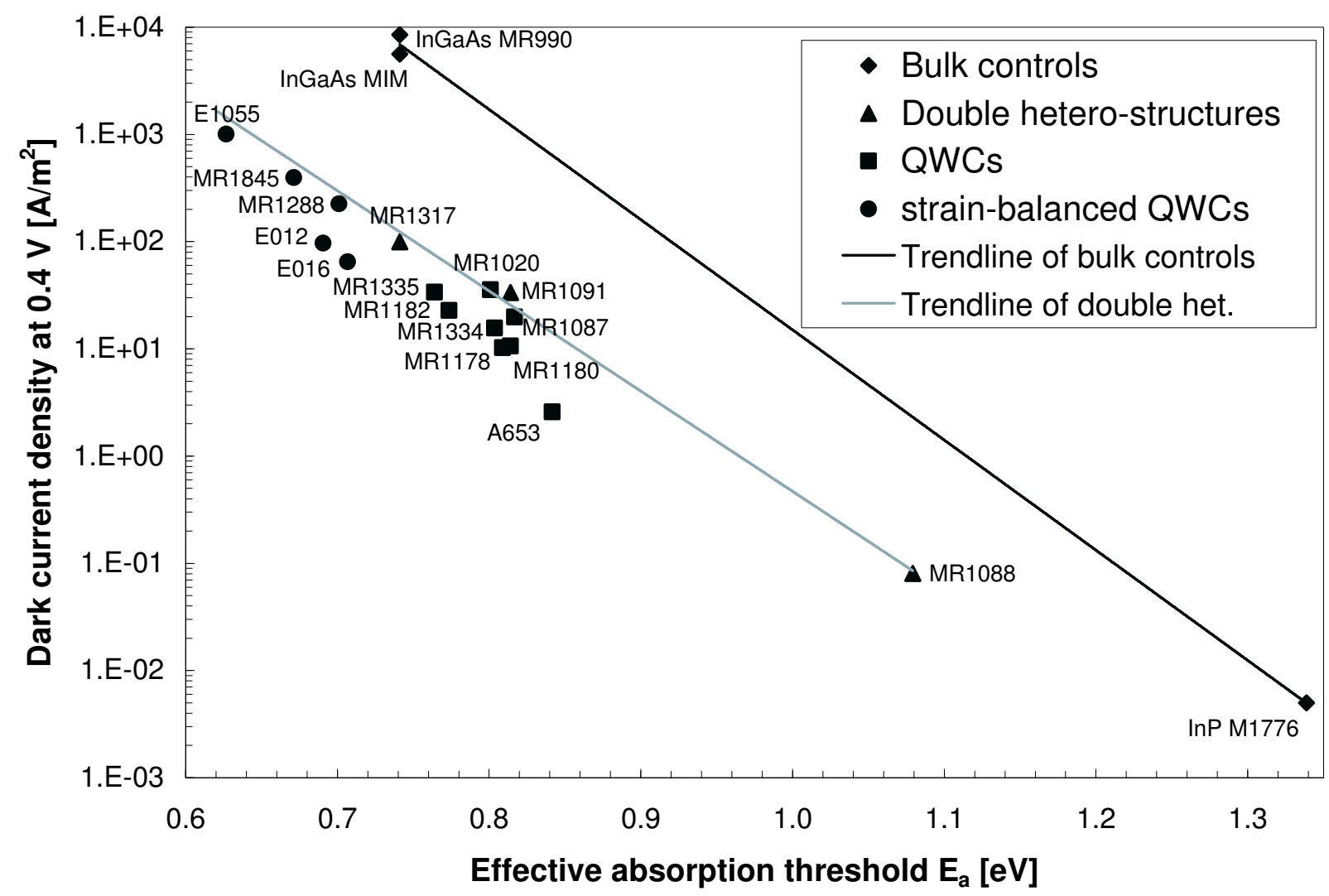

FIG. 4: Dark current density at a fixed bias of $0.4 \mathrm{~V}$ vs. absorption threshold.

TABLE III: Light I-V characteristics under $3000 \mathrm{~K}$ black-body illumination. All samples are $1 \mathrm{~mm}$ diameter mesas with $600 \mu \mathrm{m}$ diameter optical window and the cap is left on with no AR coating.

\begin{tabular}{lcccc}
\hline \hline Sample & $V_{\mathrm{oc}}[\mathrm{V}]$ & $I_{\mathrm{sc}}[\mu \mathrm{A}]$ & Fill Factor & Power Output $[\mu \mathrm{W}]$ \\
\hline QWC MR1178 & 0.477 & -109 & 0.699 & 36 \\
Well control MR1091 & 0.455 & -108 & 0.712 & 35 \\
QWC MR1180 & 0.491 & -97.0 & 0.727 & 35 \\
Barrier control MR1088 & 0.681 & -59.3 & 0.803 & 32 \\
\hline \hline
\end{tabular}

(Table III). This is mainly due to an enhancement in $V_{\text {oc }}$ compared to the well control, and a much better $I_{\mathrm{sc}}$ compared to the barrier control. However, the fill factor of MR1178 is slightly inferior compared to both controls. It should be noted that these numbers are for comparison relative to each other.

The absolute performance can be much improved by employing an appropriate grid design 
and optimising the front layers (e.g. etching off the cap layer in the optical window) and applying AR coats. In a TPV system, the illuminating spectrum and the spectral response of the cells should be matched to increase the efficiency. The system efficiency can be further improved for example by employing a mirror at the back of the device to reflect photons which have not been absorbed such as low-energy photons back to the source. Such a mirror is particularly advantageous for QWCs because the QE of the QWs is increased as photons have the chance of a second pass through the QWs.

\section{B. Strain-balanced InGaAs/InGaAs QWCs}

For TPV applications, there is increasing interest in longer wavelengths selective emitters, for example based on Thulium and Holmium, in order to match lower temperature blackbody spectra and hence achieving higher system efficiencies compared to Erbia and Ytterbia $[7,12]$. Burning fossil fuels at lower temperature at around $1500 \mathrm{~K}$ reduces $\mathrm{NO}_{x}$ emissions.

For such applications low band-gap material is required to match the long wavelength selective emitter. However, appropriate and inexpensive substrates of the required lattice constant and band-gap are not readily available, so the lower band-gap material is often strained. If the material exceeds a critical thickness dislocations are introduced which increase non-radiative recombination. In multi quantum well (MQW) systems one can avoid this strain-relaxation by strain-compensating the layers; alternating wells and barriers have bigger and smaller lattice-constants, but each period is lattice-matched to the substrate. Differences in elastic constants may need to be taken into account to minimise the stress $[3,4]$. Using this method, it is possible to obtain smaller effective band-gaps in the quantum wells than the lattice-matched material, and hence extend the absorption towards longer wavelengths without introducing excess dislocations. As long as each individual layer remains below the critical thickness, in principle an unlimited number of periods can be grown without introducing any dislocations.

However, this technique is limited by the formation of strain-balance defects [13, 14]. They are caused by the onset of undulations (wavy growth) which originate in the tensile barrier layers. The maximum number of defect free periods in an InGaAs/InGaAs straincompensated MQW is proportional to the inverse of the elastic energy density per unit area stored in each period $[13,15]$. 


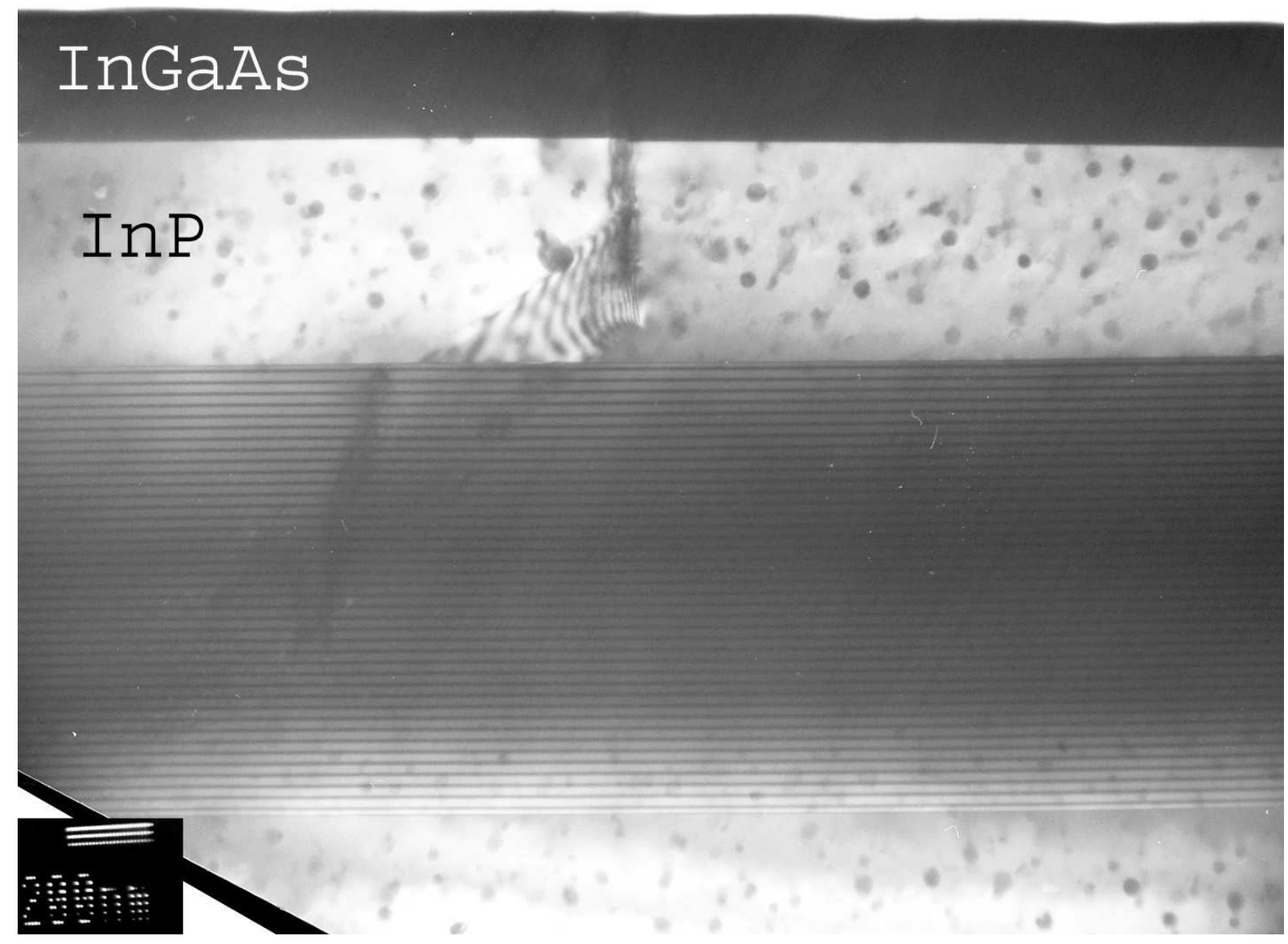

FIG. 5: Cross-sectional transmission electron micrograph of strain-compensated QWC E1055 (see Table II).

Several strain-compensated samples have been grown successfully, and one of these sample (E1055) is described in Table II. This sample has a high indium content of about $74 \%$ in the QWs, in order to extend the absorption to about $1.95 \mu \mathrm{m}$ [16], but the high In fraction also results in a high misfit of about $1.4 \%$. The barriers, by contrast, were designed to be much wider to reduce the misfit to about $-0.47 \%$. This way undulations and strain-balance defects could be avoid as is evident from the transmission electron micrograph in Fig. 5 .

Several strain-compensated QWCs are also included in Fig. 4 showing that even in the case of strain-compensation the dark current density is lower than expected from the absorption threshold. The line of expected performance as indicated in Fig. 4 is extrapolated from the results of lattice-matched bulk devices, as for these absorption thresholds no latticematched bulk material is available. This shows that with strain-compensation one can extent the absorption and at the same time it gives excellent results for strain-compensated QWCs, 
having similar or better voltage performance than expected based on the trends in the bulk material in Fig. 4.

\section{CONCLUSIONS}

We have shown that lattice-matched InGaAsP QWCs are very well suited for TPV applications such as with Erbia selective emitters. QWCs with the same effective bandgap, i.e. lower bulk bandgap, than a bulk control cell show a better voltage performance. Hence a QWC can lower the dark current, enhance the $V_{\text {oc }}$ and hence improve the efficiency compared to a bulk control cell. A major advantage of QWCs is that the bandgap can be engineered by changing the well thickness and varying the composition to fit the illuminating spectrum. This is relatively straight-forward in the lattice-matched InGaAsP system, but it can be extended to longer wavelengths by using strain-compensation techniques, achieving bandgaps that cannot be achieved with lattice-matched bulk material. We have shown that even such strain-compensated QWCs have voltage performances that are at least as good as, if not better than expected. Strain-compensated QWCs are promising candidates for TPV applications, for example with Thulia and/or Holmia selective emitters.

\section{Acknowledgments}

This research benefited from the financial support by the UK Engineering and Physical Science Research Council (grant GR/L50471) and the European Commission (grant ERK6CT-1999-00019).

[1] K. W. J. Barnham and G. Duggan, J.Appl.Phys 67, 3490 (1990).

[2] N. Ekins-Daukes, K. Barnham, J. Connolly, J. Roberts, J. Clark, G. Hill, and M. Mazzer, Appl.Phys.Lett 75, 4195 (1999).

[3] N. Ekins-Daukes, K. Kawaguchi, and J. Zhang, Crystal Growth \& Design 2, 287 (2002).

[4] C. Rohr, J. P. Connolly, N. Ekins-Daukes, P. Abbott, I. Ballard, K. W. Barnham, M. Mazzer, and C. Button, Physica E: Low-dimensional Systems and Nanostructures 14, 158 (2002).

[5] T. J. Coutts, Renewable and Sustainable Energy Reviews 3, 77 (1999). 
[6] K. Barnham, J. Connolly, and C. Rohr, eds., Special issue on thermophotovoltaics, vol. 18 of Semiconductor Science and Technology (Institute of Physics Publishing, Bristol, UK, 2003).

[7] A. Licciulli, D. Diso, G. Torsello, S. Tundo, A. Maffezzoli, M. Lomascolo, and M. Mazzer, Semiconductor Science and Technology 18, S174 (2003).

[8] J. P. Connolly and C. Rohr, in Special issue on thermophotovoltaics, edited by K. Barnham, J. Connolly, and C. Rohr (Institute of Physics Publishing, Bristol, UK, 2003), vol. 18 of Semiconductor Science and Technology, pp. S216-S220.

[9] A. Zachariou, K. W. J. Barnham, P. Griffin, J. Nelson, C. C. Button, M. Hopkinson, M. Pate, and J. Epler, in Proc. 25th IEEE PV Specialists Conference (IEEE, USA, 1996), pp. 113-116.

[10] K. W. J. Barnham, J. Connolly, P. Griffin, G. Haarpaintner, J. Nelson, E. Tsui, A. Zachariou, J. Osborne, C. Button, G. Hill, et al., J.Appl.Phys 80, 1201 (1996).

[11] N. S. Fatemi, D. M. Wilt, P. P. Jenkins, V. G. Weizer, R. W. Hoffman, Jr., C. S. Murray, D. Scheinman, D. Brinker, and D. Riley, in Proc. 26th IEEE PV Specialists Conference (IEEE, USA, 1997), pp. 799-804.

[12] M. Mazzer, A. De Risi, D. Laforgia, K. Barnham, and C. Rohr, in Proc. Society of Automotive Engineers 2000 World Congress (Society of Automotive Engineers, USA, 2000), SAE Technical Paper Series, p. 991, March 2000 in Detroit, Michigan, USA.

[13] L. Nasi, C. Ferrari, L. Lazzarini, G. Salviati, S. Tundo, M. Mazzer, G. Clarke, and C. Rohr, Journal of Physics: Condensed Matter 14, 13367 (2002).

[14] S. Tundo, M. Mazzer, L. Nasi, L. Lazzarini, G. Salviati, C. Rohr, P. Abbott, D. B. Bushnell, K. W. J. Barnham, G. Clarke, et al., Journal of Applied Physics 94, 6341 (2003).

[15] L. Nasi, C. Ferrari, L. Lazzarini, and G. Clarke, Journal of Applied Physics 92, 7678 (2002).

[16] P. Abbott, C. Rohr, J. P. Connolly, I. Ballard, K. W. Barnham, R. Ginige, G. Clarke, L. Nasi, and M. Mazzer, in The Fifth Conference on Thermophotovoltaic Generation of Electricity, edited by T. J. Coutts, G. Guazzoni, and J. Luther (American Institute of Physics, Woodbury, New York, 2003), vol. 653 of AIP Conference Proceedings, pp. 213-221, Rome, Italy, Sept. 2002 . 